\title{
Discussion on the Teaching Mode of Computer Specialty based on the Combination of College and Enterprise
}

\author{
Ziyang Han, Shoujin Wang, and Jifei Liu \\ School of Computer Science, Shenyang JianZhu University, Shenyang 110000, China. \\ Phenixhans@QQ.com, 23917240 @ QQ.com, 5998587@ QQ.com
}

\begin{abstract}
The study of Computer Science in Colleges and universities, attaches great importance to the theory, but attaches little to the practice, teaching methods are still very traditional, theoretical study and social practice out of touch, the results of school education and social needs of the fault. Adopting the teaching mode of the combination of school and enterprise, the society needs to enter the school, and the school and the enterprise can train the practical students together. United have some well-known enterprises, select the actual software development projects suitable, organize students to participate in the project development, learning development, team awareness, communication form, management methods, and get experience from the students, change the traditional classroom learning. Practice has proved that this method can better enable students to complete the course of learning, master the development of practical experience, for students to work smoothly laid the foundation.
\end{abstract}

Keywords: Computer specialty; Teaching model; Coalition of college and enterprise; Students practice

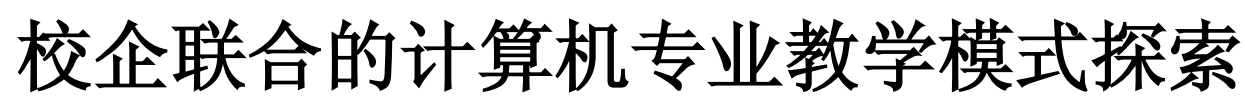

\author{
韩子扬，王守金，刘继飞 \\ (沈阳建筑大学 信息与控制工程学院 计算机系, 辽宁 沈阳 110168)
}

摘要: 普通高校的计算机专业学习, 重理论, 轻实践, 教学方法仍然很传统, 理论学习与社会实践脱节, 学校教育结果 与社会需求出现断层。采用校企联合的教学实践方式, 让社会需求进入学校, 学校与企业共同培养切合实际的学生。联合具 有一定知名度企业, 选取合适的实际软件开发项目, 组织学生参与项目开发, 学习其中的开发方式, 团队意识, 交流形式, 管理方法, 并从中获取实践经验, 改变学生传统的课堂学习方式。实践证明, 该方法能更好的让学生完成课程的学习, 掌握 开发实践经验, 为学生能顺利的走向工作岗位奠定了基础。

关键词: 计算机专业; 教学改革; 校企联合; 学生实践

中图分类号: G646 文献标志码: A

引言

随着移动电子设备性能的不断提升, 网络建设不断完善, 带动电子信息产业迅猛发展, 各行各业竞相 进入电子信息化时代, 新的理念不断推出, 方便快捷的电子应用工具遍布我们生活的方方面面, 随之而来 的就是对专业人才的巨大需求量。但是另一方面数据显示, 普通高校的计算机专业毕业生就业情况却不容 乐观, 大量应届毕业生没有按时就业。中国软件协会称, 现阶段我国软件开发基础人才不足, 已成为制约 我国软件产业发展的瓶颈。为什么会出现这样的问题? 分析其原因发现, 虽然多数高校都设置了计算机专 业, 但是由于课程结构设置原因与教学方法问题, 使得学生在学习过程中重视理论学习, 重视考试, 课堂 教育占据学生主要学习时间, 学生缺乏动手参与过程, 没有实践经验, 致使计算机专业学生在毕业时, 实 
际工作能力与社会实际需求衔接不上，学生找不到合适的工作，企业找不到合适的人才。

十三五规划中，对于高校办学提出了新的指导方向，在教育方式上，“推进产教融合、校企合作，推 行工学结合、校企合作的技术技能人才培养模式”, 改变了以往的课堂教学思路, 让学生走出课堂, 社会 企业走进学校, 在实践中融合专业与就业之间的关系, 缩短学生与工作岗位之间的距离, 借助社会力量补 充学校教育的不足, 延展传统教学。

\section{1 计算机专业教育现状}

计算机发展史仅有几十年, 却在现代生活中, 占据了至关重要的位置, 工作内容划分也变得越来越细 致。特别是在与其他专业相结合的跨专业领域应用, 是计算机发展的主要方向。

目前, 高校对计算机类课程采用的教学方式是: 以理论讲解为主、实践为辅, 课程的考核依靠传统的 卷纸考试, 靠分数定成绩。但是作为计算机课程内容的特点: 概念多、图表多、理论多、文档多、系统性 强, 涉及面广, 缺少实践环节, 学生接受起来比较困难, 很难将众多的知识点联系起来, 更不会运用它来 解决实际问题。有的高校在教学中引入课程设计, 但这种实践终究是跟社会需求是脱节的, 它与实际的项 目开发相比, 缺少必要的细节分析和实际可操作过程, 像可行性研究、需求分析这样的内容 ${ }^{[1]}$, 没有经过 实际的动手操作, 是不会了解其中的关联关系, 也就不会应用计算机去开发与设计 ${ }^{[2]}$ 。另外, 值得我们注 意的是, 在众多优秀的计算机类教材中, 很少提及如何指导学生实践内容。高度抽象化课程内容和教条的 理论, 使学生不知道如何去进行实践。

\section{2 教学改革的方法探索}

依靠传统的学校课堂教育, 已经不能解决学生缺乏实践经验的问题, 必须寻找相应的教学改革方法, 在校外, 软件企业在开发过程中完成了大量的项目, 具备成熟的开发经验和系统化的文档, 很多知名企业 完成了标准化的认证, 在内外都享有较高的声誉, 联合这样的软件企业, 并结合企业实际已完成的项目, 在学生具备了软件开发基础后, 根据企业项目实际需求和开发背景, 以软件开发人员与学校教师联合对学 生进行软件开发实践指导 $[3,4]$, 会改善学生的学习效果。

\section{1 采用校企联合方式的主要优势}

1) 直接将课程理论与开发实践结合起来, 既能强化理论学习, 又能使理论得到了应用, 真正做到学 以致用。

2) 学生在学校学习期间, 就可以体验企业中的工作方法和环境氛围, 可以加深学生对专业前景的认 识。

3) 学生在完成课程学习及项目实践后, 具有了实际项目的开发经验, 提高了学生的实际动手能力, 这一点对学生以后的就业十分有帮助 ${ }^{[5]}$ 。

\section{2 计算机教学改革的实施步骤}

以校企联合方式, 开展计算机教学与实践改革方法, 可以将计算机课程学习和项目实践分成 5 个阶段 ${ }^{[6]}$ 。

第一阶段: 在校期间理论学习期间, 需要经过 4-5 个学期, 教师完成计算机理论教学内容, 学生具备 基础知识, 对专业有一定认知, 对从事行业有所了解。教师在这一阶段重点强化学生的理论知识, 编程工 具的学习, 使学生了解软件项目开发是一个规范化, 标准化和系统化的过程, 它具有典型的开发方法可以 
遵循 ${ }^{[7]}$ 。

第二阶段: 项目需求分析培训。这一阶段需要聘请软件企业的项目开发人员作为课程实践的指导教师, 选取企业的项目案例, 对其中的需求进行调整, 使项目的规模和难度适合学生的能力; 指导学生做需求分 析，将需求分析的过程融入到实践当中。聘请软件企业的项目经理做指导教师的原因是：项目经理拥有实 际项目开发的经验和管理策略, 以及在处理实际问题时所采用的方法, 这些经验和方法是在校教师所无法 具备的, 正是因为这一点, 项目开发人员的实践经验要比在校理论教师更有说服力 ${ }^{[8]}$ 。

第三阶段：项目文档规范培训。学生在学习软件开发过程中, 由于教材几乎不涉及到系统化的文档模 板, 学生在实践应用中很少编写文档, 或者编写不出有实际意义的文档 ${ }^{[9]}$ 。而大多数软件企业的文档是经 过长时间积累形成的, 对外界是保密的, 所以对企业的原有文档进行改造, 这一点对于企业来说非常必要; 去掉软件企业文档特殊化的内容和需要保护的内容, 既保护了企业的商业秘密, 也使文档适合学生学习。 规范化的和系统化的文档书写和编码规范, 使学生顺利完成项目的基础。

第四阶段: 学生开发项目实践。教师需要根据学生的个人实际能力和对开发的理解, 将学生分成项目 小组 ${ }^{[10]}$, 每小组成员不超过 6 人, 控制项目开发周期在 2-4 周左右, 这个时间对于项目实践是比较充分的, 具体分工如下:

（1）项目模块负责人 1 人，负责文档的设计，协调组内分工，与教师和项目经理沟通，组内进度控 制;

（2）项目模块分析与设计 2 人，完成功能划分，细化模块内容;

（3）代码开发不超过 2 人，完成规范化代码设计，完成适度的注释;

(4) 项目测试 1 人。

教师和项目经理 ${ }^{[11]}$ 作为项目的总负责人的分工如下:

项目经理：对小组进行项目介绍，组织需求分析；组织学生学习企业化的管理方法，制定规章制度， 负责学生在项目分析上存在的问题, 解决实现技术上的难题; 进行团队合作指导, 进行项目总体进度控制; 在沟通方法上，与每个项目小组负责人进行沟通，不直接与组员沟通。

授课教师：负责理论指导，制定进度计划，进行项目分组及人员安排与调整。

第五阶段: 成绩考核。放弃传统的单一考试方式, 采用多方面的综合考核方式, 既要考查学生是否学 习到了理论知识, 又要考查是否获取了实践经验, 需要从 4 个方面考虑。

1）理论学习考核: 由授课教师完成考核, 以考试为主的理论成绩比例, 在此应该大幅度降低;

2）文档考核：由项目经理考核，文档是项目实践的基础，同时也是项目质量的保证，重点需要考核 文档的阶段性与文档的规范性;

3) 程序考核: 由项目经理考核, 程序是否能够正常运行, 代码是否规范等, 按照需求分析的文档测 试, 是否符合需求分析;

4）项目答辩：由授课教师和项目经理共同考核，既要考查学生的现场应变能力，又要挖掘学生在实 践中的得失; 既让学生在学习过程中进行自我总结, 又要听取学生在学习后的效果反馈, 以改善后续的实 践方法。

综合以上的实施过程, 实现改革过程的示意图, 如图-1: 


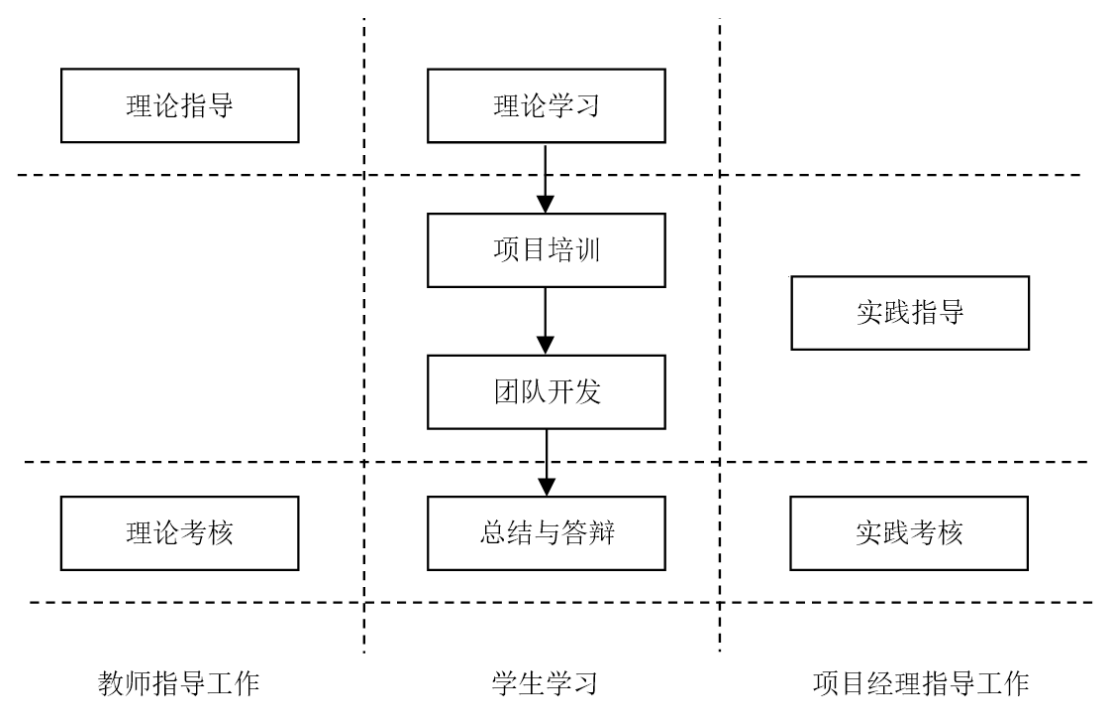

图 1 计算机专业实践实施步骤

\section{3 在实践过程中的注意事项}

1）培养团队合作意识, 发挥集体的力量。在学习程序设计的时候, 是以个人的方式学习, 但是在项 目开发阶段, 单靠个人能力是不行的, 所以, 养成良好的团队精神, 加强沟通, 是项目顺利开展的前提。

2) 以文档为开发依据, 养成以文档为软件驱动的开发思想。学生对软件开发的学习是从编写代码开 始的, 致使学生对文档的认识较少, 忽略了文档的重要性, 应该让学生认识到, 文档本身就是软件产品。 没有文档支撑的软件, 就不能称为软件。强调软件文档的编制在软件开发工作中占有突出的地位和相当的 工作量。文档的分发，回收，归档管理，按时审查文档是保证软件项目质量的重要手段。

3）重视管理的规范性。学生在校期间, 接受学校的管理制度, 与企业有很大不同, 企业严格的规章 制度是软件企业生存和发展的重要保障, 让学生认识到不同的管理制度对于专业的影响, 可以使学生尽早 具备严谨的工作态度和习惯, 例如: 编写可读性好的代码, 是为了便于别人看懂, 更重要的是, 代码容易 复查, 减少错误出现的几率, 便于修改调试, 同时也提高了代码的外在质量。可读性好的代码并不比含糊 的代码多花时间, 运行时间相差也微乎其微, 让学生培养良好的编程习惯, 更重要的是在团队成员间建立 了配合上的默契。

4）尊重开发进度, 制定切合实际的开发计划。在学生进行项目开发过程中, 常常会遇到的执行进度 上的问题有如下两种:

（1）软件的开发进度会拖延, 实际进度比预期进度拖延也不意外。为了解决这个问题, 我们要设计 完整的进度计划, 在计划中既要详细的安排工作内容, 又要为工作的复查留有充足的时间, 让学生充分认 识到在工作中可能遇到的问题。

(2) 对学生已经完成的工作, 存在不满意或者不正确的地方, 不能视而不见, 因为软件开发中, 错 误是不可避免的, 正确指导学生认识错误的来源; 初次接触软件开发的学生, 会有疲于繁琐管理的心态, 过度追求进度很可能会导致错误的发生, 要认真的指导学生找到错误的根源, 减少错误发生几率。

为了避免学生在项目团队开发过程出现任务的推脱和懒散的情况, 需要项目进度计划详细到每个半天 
的工作内容, 所有的任务都需要有明确的分割界限, 所有的任务必须落实到个人。使学生认识到每个成员 都是项目的一部分，树立对项目的责任心。

5）及时总结, 及时改进, 失败的经验与成功的应验同样有用。根据学生的能力不同, 不能以同样的 标准来考查学生。有学生可能没有完成项目任务, 导致项目失败, 这种情况下, 指导学生总结失败的经验 和教训, 这种收获, 可能比成功后短暂的喜悦更重要, 它让学生知道了哪些过程中存在问题, 哪些过程还 需要改进, 这样的总结是最好的学习方法, 也会让学生把经验和教训铭记在心。

\section{3 教学效果分析}

在计算机专业教育教学改革的 3 年实践中，我们先后与多家较为知名的软件开发企业进行联合培养， 对实践的结果进行跟踪, 获得了较好的效果, 学生专业能力迅速提升, 软件开发企业也从中接纳优秀的学 生。在实践过程中, 适当调整理论教学时间, 大幅度增加项目实践时间, 是教学改革的关键。改革结果表 明: 项目实践增加了学生实际项目开发的经验, 学生的项目开发能力普遍提高, 在此后的学生就业过程中, 更为学生赢得了竞争力。

\section{4 结论}

在十三五教学指导思想下，传统的计算机教学模式需要新的契机，本文实验校企联合的教学方式，采 用教师教授学生理论知识，结合软件企业的项目开发人员指导学生项目实践，二者结合完成计算机的课程 学习, 实现了把理论融入实践, 在实践中学习理论的教学模式, 学生从课堂教学模式中解放出来, 具有针 对性的学习软件开发的方法, 项目管理的经验, 学生有了实践经验, 为将来就业奠定了基础。此外, 这种 有针对性的课程教学模式也适合相关专业的课程改革。

\section{参考文献:}

[1] 牛长海. 中原经济区建设视阈下河南高校实践教学模式探讨 $[\mathrm{J}]$. 东北师大学报（哲学社会科学 版), 2012, (3) : 195-198.

[2]杨海峰,刘同冈. 基于校企联合的本科毕业设计指导模式探讨 $[\mathrm{J}]$. 教育教学论坛, 2015, (8): 37-38.

[3] 赵大兴, 孙国栋, 王璜等. 地方本科院校校企联合培养工程应用型人才模式探讨 [J]. 教育教学论 坛, 2016, (29) : 170-171.

[4]龚建荣, 阙善材. 应用型本科院校校企合作人才培养模式探讨 $[J]$. 开封教育学院学报, 2016, 36 (10) : 95-96.

[5]朱东弼, 许一男, 马勇虎等. 电子信息类专业校企联合培养机制构建 $[\mathrm{J}]$. 中国教育技术装备, 2015, (22) : 108-109.

[6]梁佩荣, 林洁丽, 曹辉等. 校企联合的应用型人才培养平台建设探索 [J]. 产业与科技论坛, 2014, (17) : 255-256.

[7]付士军,刘志强. 基于校企联合地方工科大学生实践教学模式研究 [J]. 科技信息, 2014, (8) : 38 .

[8]王新荣, 李小海, 龙泽明等. 专业学位硕士研究生校企联合培养的探索与实践 [J]. 中国电力教育, 2014, (8) : 16-17.

[9] 石荣丽, 阮涃静. 校企联合高层次应用型物流管理人才培养模式探究 $[J]$. 黑龙江教育 (高教研究与评估 版), 2015, (3) : 70-71.

[10]包丽, 张洪军, 李西兵等. 校企联合探索实验教学新模式 [J]. 实验技术与管理, 2014, (6) :200-202, 212.

[11] 李建荣. 基于校企联合、面向企业需求的高校物联网专业毕业设计模式初探 [J]. 电子测试, 2015, (22) : 152-153.

\section{Reference:}

[1] Niu Changhai on the Practical Teaching Mode of Henan Colleges and Universities from the 
Perspective of the Construction of Zhongyuan Economic Zone [J]. Journal of Northeast Normal University (Philosophy and Social Sciences), 2012, (3): 195-198.

[2] Yang Haifeng, Liu Tonggang Discussion on the Guidance Mode of Undergraduate Graduation Design Based on the Combination of School and Enterprise [J]. Education and Teaching Forum, 2015, (8): 37-38.

[3] Zhao Daxing, Sun Guodong, Wanghuang, etc. Discussion on the Mode of Cultivating Applied Talents in Local Colleges and Universities [J]. Education and Teaching Forum, 2016, (29): 170-171.

[4] Gong Jianrong, Que Shancai Discussion on the Mode of Cultivating Talents of School Enterprise Cooperation in Application - oriented Universities [J]. Journal of Kaifeng Institute of Education, 2016, 36 (10): 95-96.

[5] Zhu Dongbi, Xu Yinan, Ma Yonghu, etc. Construction of Joint Training Mechanism of School and Enterprise for Electronic Information [J]. China Education Technology \& Equipment, 2015, (22): 108-109.

[6] Liang Peiying, Lin Lijie, Caohui, etc. A Probe into the Construction of the Training Platform for the Application of Talents in the Combination of Enterprise and Enterprise [J]. Industry and Technology Forum, 2014, (17): 255-256.

[7] Fu Shijun, Liu Zhiqiang A Study on the Practical Teaching Mode of University Students in Colleges and Universities [J]. Technical Information, 2014, (8): 38.

[8] Wang Xinrong, Li Xiaohai, Liu Zeming, etc. Exploration and Practice of Joint Cultivation of Master and College Graduates [J]. China Electric Power Education, 2014, (8): 16-17.

[9] Shi Rongli, Ruan Xianjing A Probe into the Training Mode of High - level Applied Logistics Management in Colleges and Universities [J]. Heilongjiang Education (Higher Education Research and Evaluation), 2015, (3): 70-71.

[10] Baoli, Zhang Hongjun Li Xibing, etc. A New Model of Experimental Teaching from School enterprise Joint [J]. Experimental Technology and Management, 2014, (6): 200-202,212.

[11]Li Jianrong On the Graduation Design Pattern of the Internet of Things in Colleges and Universities Based on the Combination of Enterprise and Enterprise and the Enterprise Needs [J]. Electronic Testing, 2015, (22): 152-153. 